PAPER

\title{
Acute small subcortical infarctions on diffusion weighted MRI: clinical presentation and aetiology
}

\author{
T Seifert, C Enzinger, M K Storch, G Pichler, K Niederkorn, F Fazekas
}

J Neurol Neurosurg Psychiatry 2005;76:1520-1524. doi: 10.1136/jnnp.2005.063594

See end of article for authors' affiliations

\section{Correspondence to:}

Dr Thomas Seifert,

Department of Neurology,

Graz Medical University,

Auenbruggerplatz 22, A-

8036 Graz, Austria;

thomas.seifert@

meduni-graz.at

Received 16 January 2005

Revised version received

21 March 2005

Accepted 21 March 2005
Objective: To determine the clinical presentation and aetiology of small subcortical infarctions as found on diffusion weighted magnetic resonance imaging (DWI). DWI is both sensitive and specific in the early detection of acute ischaemic brain lesions irrespective of pre-existing vascular damage.

Methods: Ninety three patients were identified showing subcortical or brainstem DWI lesions $<1.5 \mathrm{~cm}$ in diameter within a maximum of 7 days from the onset of stroke symptoms. The patients' clinical status on admission was reviewed according to the Oxfordshire Community Stroke Project (OCSP). The results of procedures searching for cerebrovascular risk factors, large artery disease, and potential sources of cardiac embolism were included to determine stroke aetiology. Magnetic resonance imaging scans were also reviewed for concomitant changes that could support the aetiologic classification.

Results: Only 41 (44.1\%) patients presented clinically with a lacunar syndrome according to OCSP criteria. The nine $(9.7 \%)$ patients who showed two or more DWI lesions in different vascular territories were also significantly more likely to have potential sources of cardiac embolism $15 / 9,55.6 \%$ v 20/84, 23.8\%). Hypertension was significantly more prevalent in the group of patients who showed a microangiopathy related imaging pattern, but this pattern did not exclude the presence of large artery disease or a possible cardioembolic source of stroke.

Conclusion: Identification of small subcortical infarctions as the cause of stroke appears quite uncertain based on clinical characteristics only. DWI adds significant aetiologic information but does not obviate the search for other potentially causative mechanisms.
S mall subcortical infarctions are currently assumed to have several aspects in common as proposed by the concept of "lacunar stroke". ${ }^{1}$ First, histopathologic studies found that ischaemic subcortical lesions $<1.5 \mathrm{~cm}$ in diameter result predominantly from occlusion of small penetrating arteries, which is presumed to be due to microatheroma or lipohyalinosis in the penetrating vessel or due to an atheroma lining the parent vessel. ${ }^{2-6}$ Early reports also suggested that the occlusion of a penetrating artery may be due to embolism, ${ }^{35}$ but subsequent investigations could not confirm a significant contribution of cardiac embolism to lacunar infarction. ${ }^{78}$ Second, small subcortical infarctions have been linked to distinct clinical syndromes: pure motor stroke, pure sensory stroke, ataxic hemiparesis, dysarthriaclumsy hand syndrome, and sensory motor stroke. ${ }^{9}$ Third, patients suffering from lacunar stroke are considered to have a better prognosis compared to patients with non-lacunar stroke. ${ }^{1}$ As a consequence, the concept of lacunar stroke has entered stroke classifications such as that proposed by the Oxfordshire Community Stroke Project $(\text { OCSP })^{10}$ or criteria used for the description of stroke aetiology and subsequent patient selection for treatment trials such as the TOAST (Trial of Org 10172 in Acute Stroke Treatment) criteria. ${ }^{11}$

The assumptions mentioned above were derived from clinico-pathological correlations and studies that used computerised tomography for cerebral imaging, which is rather insensitive for detecting small subcortical lesions, at least in the acute stage. The advent of magnetic resonance imaging (MRI) markedly improved this situation. However, focal signal abnormalities similar to acute lesions may be seen on MRI in a variety of clinical settings and even with normal ageing. This often complicates the delineation of acute subcortical infarcts especially in the presence of preexisting tissue damage. ${ }^{12}{ }^{13}$ In this context, the introduction of diffusion weighted MRI (DWI) has been a further significant step forward. Restricted diffusion is typically seen only during the first few days of infarction and thus allows a good separation of acute from old lesions including chronic white matter damage. ${ }^{14}$ The addition of DWI to standard MRI protocols not only enables identification of the acute, clinically relevant lesion but may also provide aetiologic insights. The detection of multiple acute subcortical ischaemic lesions by DWI has been considered to be suggestive of embolism. ${ }^{15-17}$ This view was challenged recently by a report proposing that diffuse damage to small vessels might also cause multiple subcortical DWI lesions. ${ }^{18}$

In view of the potential of DWI for acute stroke imaging, we have attempted to collect further information regarding the concepts of lacunar stroke by studying consecutive patients who presented with an acute neurological deficit and were shown to have small subcortical infarcts on DWI. This was prompted primarily by (a) the observation of a frequent clinical misclassification of these lesions and (b) uncertainties regarding the aetiologic implications of finding one or more small subcortical ischaemic lesions in relation to an acute stroke syndrome.

\section{METHODS}

We identified 1304 acute ischaemic stroke patients who were admitted to our hospital between August 2001 and December 2003. Brain MRI including DWI was performed on these patients when their clinical condition was stable enough to

Abbreviations: DWI, diffusion weighted magnetic resonance imaging; ECG, electrocardiogram; ICA, internal carotid artery; LACS, lacunar syndrome; MRI, magnetic resonance imaging; OCSP, Oxfordshire Community Stroke Project; PACS, partial anterior circulation syndrome; POCS, posterior circulation syndrome; TACS, total anterior circulation syndrome; TEE, transoesophageal echocardiography; TOAST, Trial of Org 10172 in Acute Stroke Treatment; TTE, transthoracic echocardiography; WMH, white matter hyperintensities 
undergo examination, when there were no contraindications to MRI (for example, a cardiac pacemaker), and the patient agreed to scanning. The respective case records were screened for patients who fulfilled the following criteria: neurologic deficit present on admission and persisting for at least $24 \mathrm{~h}$, MRI performed between 0 and 7 days after the onset of clinical symptoms, and subcortical or brainstem DWI lesions $<1.5 \mathrm{~cm}$ in diameter. Patients with additional cortical involvement on DWI were not included. Based on these criteria, we identified a total of 93 patients. The clinical examination of patients on admission was documented in a standardised scheme according to predefined in-house guidelines. A primary diagnosis of vascular territory involved, that is, anterior, median, posterior cerebral artery, or basilar artery, was made based on the clinical examination. For our retrospective study, an investigator (CE) who was blinded to the patients' age, gender, complaints, history, cerebrovascular risk factors, the primary diagnosis of vascular territory, cerebral imaging, and all other results from diagnostic procedures, reviewed the documented clinical examinations and classified the patients according to the OCSP. ${ }^{10}$

DWI was performed as part of a routine MRI protocol for stroke patients on a $1.5 \mathrm{~T}$ system (Gyroscan NT, Philips Medical Systems, Eindhoven, The Netherlands) as follows: TR 4600, TE 81, slice thickness $5 \mathrm{~mm}$, gap $1.0 \mathrm{~mm}$, number of excitations (NSA) 2, field of view (FOV) $200 \mathrm{~mm}$, rectangular field of view (RFOV) $100 \%$, matrix $128 \times 128$. All scans were reviewed by a trained neurologist with MRI experience (TS) unaware of the clinical or other information. Cerebral white matter hyperintensities (WMH) on conventional MRI were rated using a modification of the Fazekas scale ${ }^{19}$ which was developed recently as part of a multinational European study on age related white matter changes ${ }^{20}$ as follows: no WMH (grade 0 ), punctate (grade 1), early confluent (grade 2 ), and confluent (grade 3) WMH.

We further attempted to define imaging criteria that might serve to better separate true microangiopathy related cases of subcortical infarction from those of other possible aetiologies. We thus defined a microangiopathy related imaging pattern to consist of the presence of only a single lesion on DWI plus grade 2 or $3 \mathrm{WMH}$ and no previous infarctions other than lacunar on conventional MRI (fig 1). Conversely, all other patients, that is, patients who showed multiple lesions on DWI, or grade 0/1 WMH, or previous non-lacunar infarctions, were felt to show a pattern not suggestive of microangiopathy (fig 2).

The following vascular risk factors were identified as defined by preadmission history or the need for medication at discharge: hypertension, hypercholesterolaemia, and diabetes mellitus. Cigarette smoking of at least 1 pack year (a vascular risk factor) within the past 5 years was recorded. Duplex sonography of carotid and vertebral arteries, transcranial Doppler sonography, and electrocardiograms (ECG) were performed in all patients. The severity of internal carotid artery (ICA) stenosis was graded according to established morphology and velocity criteria. ${ }^{21}$ TTE was performed in 80 $(86.0 \%)$ patients. Amongst these, TEE was additionally performed in 21 patients $(22.6 \%$ of all patients). Magnetic resonance angiography was carried out in 22 (23.7\%) patients, and conventional angiography in three (3.2\%) patients. Screening for serologic parameters indicating vasculitis (anti-nuclear antibodies, complement complexes, immunoglobulins) was performed in $24(25.8 \%)$ patients, and screening for coagulopathy was carried out in $30(32.3 \%)$ patients.

For statistical analysis, the $\chi^{2}$ test was used to compare risk factors between patient groups and Student's $t$ test was performed to compare differences in age between different

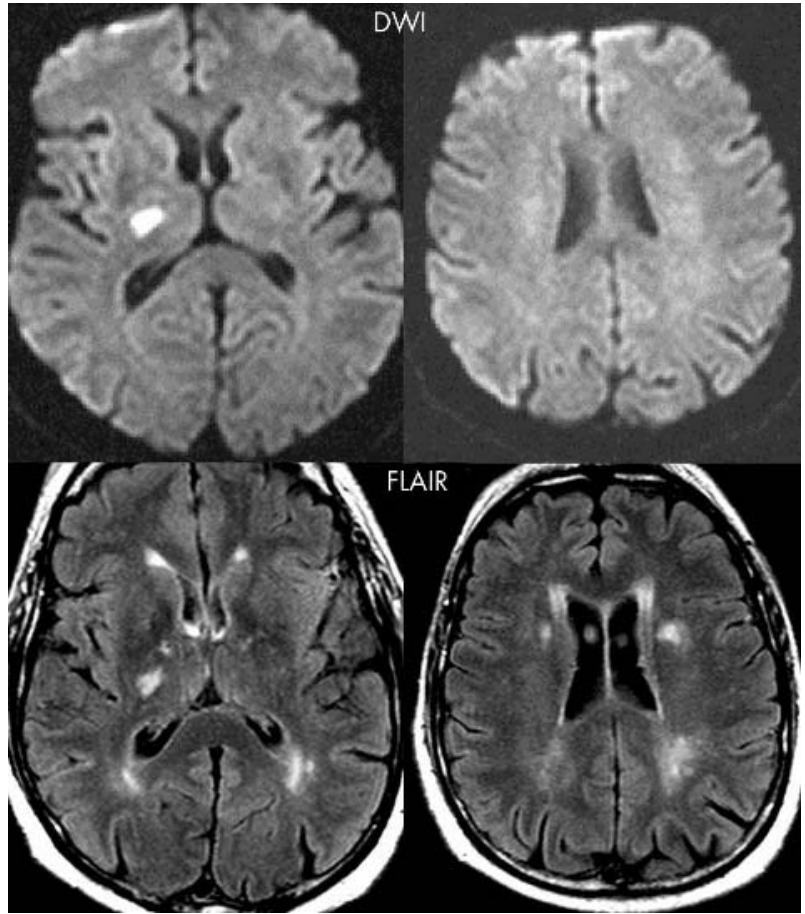

Figure 1 Microangiopathy related MRI imaging pattern (single DWI lesion plus grade $2 \mathrm{WMH}$ and no previous infarctions other than lacunar) in a 70 year old male patient presenting with a lacunar syndrome (LACS). Hypertension and smoking were identified as cardiovascular risk factors. Large artery disease was excluded by carotid duplex and transcranial Doppler sonography. Serologic parameters indicating vasculitis were normal. No coagulopathy or atrial fibrillation was found. Transthoracic echocardiography (TTE) was normal.

patient groups. A p value of less than 0.05 was considered to indicate statistically significant differences.

\section{RESULTS}

We identified 93 patients with subcortical or brainstem DWI lesions $<1.5 \mathrm{~cm}$ in diameter (63 men, 30 women; mean age 65.7 years, range $28-89)$. The lesions were located in the vertebrobasilar circulation in $57(61.3 \%)$ patients, thereby affecting areas supplied by pontine perforators in 22 patients and by thalamic, lateral medullary, and midbrain perforators in 18,10 , and 7 patients, respectively. Thirty four (36.6\%) patients had lesions in the ICA territory; 23 of these had lesions in the basal ganglia and internal capsule and 11 had lesions in the centrum semiovale. Two $(2.1 \%)$ of the 93 patients showed lesions in regions of both the anterior and posterior circulation.

A lacunar syndrome (LACS) was found clinically in 41 $(44.1 \%)$ patients, a posterior circulation syndrome (POCS) in $37(39.8 \%)$, a partial anterior circulation syndrome (PACS) in $14(15.0 \%)$, and a total anterior circulation syndrome (TACS) in one $(1.1 \%)$ patient. When patients with small subcortical infarcts located only in areas supplied by the anterior circulation $(\mathrm{n}=34)$ were considered, we found a LACS in $52.9 \%$ of patients. Patients with small infarcts in the posterior circulation $(n=57)$ showed a LACS in $40.4 \%$ of cases.

The major cerebrovascular risk factors associated with small vessel disease, that is, hypertension, hypercholesterolaemia, diabetes mellitus, and smoking, were found in $72.0 \%, 55.9 \%, 32.3 \%$, and $38.7 \%$ of all patients, respectively. No statistically significant differences in the prevalence of these vascular risk factors were found between men and women. Potential sources of cardiac embolism were found in $25(26.9 \%)$ of our patients as follows: atrial fibrillation and 


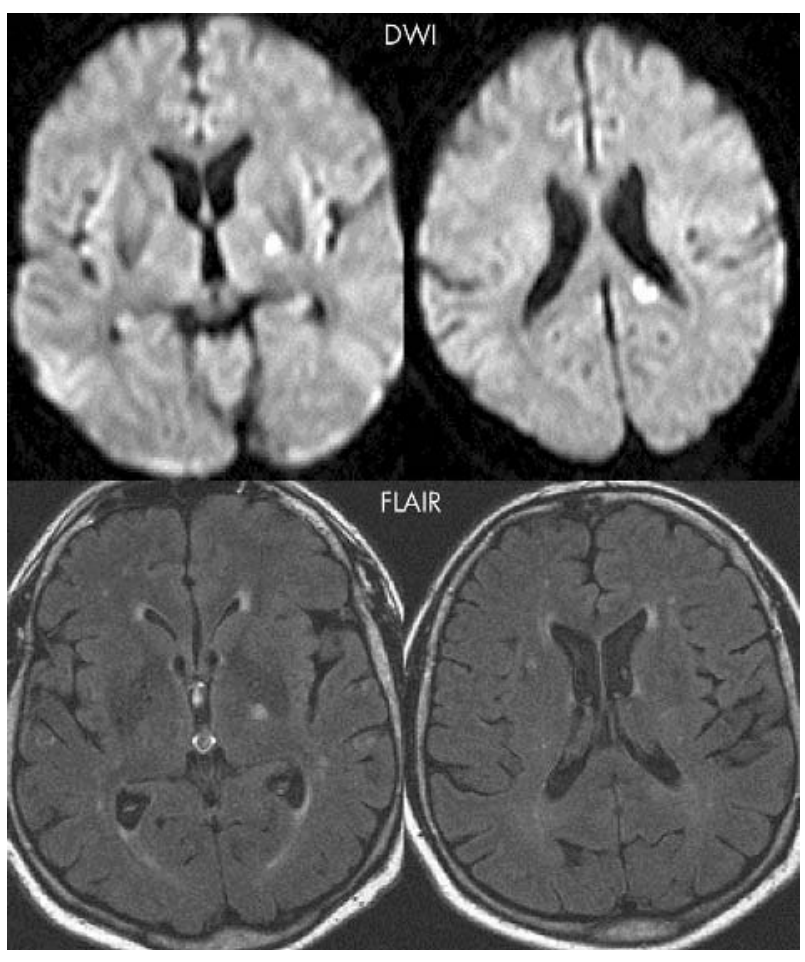

Figure 2 MRI imaging pattern not suggestive of microangiopathy (two DWI lesions, grade 1 WMH) in a 56 year old male patient presenting with a posterior circulation syndrome (POCS). There was a history of smoking, but there was no hypertension, hypercholesterolaemia, or diabetes mellitus. Large artery disease and intracranial vascular abnormalities were excluded by sonography and magnetic resonance angiography. Serologic parameters for vasculitis were negative. Coagulopathy and atrial fibrillation were excluded. Transoesophageal echocardiography (TEE) revealed a patent foramen ovale as a potential source of cardiac embolism.

atrial sludge in 12 patients, patent foramen ovale in seven, ventricular wall hypokinesia in three, ventricular thrombus in one, mitral valve prosthesis with insufficient oral anticoagulation in one, and aortic valve myxoma in one. Large artery disease was found in $25(73.5 \%)$ of 34 patients with a lesion in the ICA territory, that is, plaques or stenosis of the ipsilateral common or internal carotid artery in 24 patients and a plaque in the ascending aorta in one patient. ICA stenosis $>50 \%$ on duplex ultrasound was found in three of these patients, and intracranial ICA stenosis in one patient. In $16(28.1 \%)$ of 57 patients with a lesion in the vertebrobasilar territory, large artery disease was revealed as follows: dissection or occlusion of vertebral artery in 11 patients, subclavian steal phenomenon in two, basilar artery aneurysm in one, and plaque of ascending aorta in one.

We found two or more DWI lesions in 14 of our patients (15.1\%). Further review of DWI images revealed that in five of these patients showing two lesions each, the lesions were probably a consequence of the occlusion of the same small vessel as follows: a probable common trunc of bilateral thalamo-subthalamic paramedian arteries causing bilateral paramedian thalamic lesions ${ }^{22}{ }^{23}$ as revealed in two patients, adjacent small DWI lesions in the internal capsule in another two patients, and adjacent lesions in the pons in one patient suggesting occlusion of a single penetrating artery. The remaining nine patients (9.7\% of all patients) showed two or more DWI lesions in different vascular territories. In addition to ECG and ultrasound, all nine also had TTE and, additionally, four of them had TEE. Magnetic resonance angiography was performed in two and conventional angiography in one of these patients with normal results. Screening for serologic parameters indicating vasculitis or coagulopathy was carried out in four and five of the patients, respectively, with normal results. The characteristics of these patients in comparison to all other patients are given in table 1 . No statistically significant differences were found between these patient groups concerning gender, clinical syndrome, or vascular risk factors. Potential sources of cardiac embolism were detected significantly more often in the group of patients with multiple DWI lesions in different vascular territories.

We found 36 (38.7\%) patients with a microangiopathy related imaging pattern. These patients were significantly older, the ratio of both genders was shifted significantly towards women, and hypertension was significantly more prevalent in this group. The prevalence of other vascular risk factors, large artery disease, or potential sources of cardiac embolism was not statistically different between the groups of different imaging patterns (table 1).

\section{DISCUSSION}

Clinical examination of stroke patients is not sufficient to differentiate lacunar infarcts from other stroke subtypes. In our study, only $44.1 \%$ of patients in whom a small subcortical infarction was found on DWI presented clinically with a LACS. This is in line with earlier studies which pointed out the limitations of stroke classification by clinical criteria only. In a previous observation using DWI and perfusion weighted imaging, lacunes could also not be reliably diagnosed on the basis of clinical findings only. ${ }^{24}$ Other studies have shown that only $75 \%$ of patients are correctly classified clinically by the OCSP classification, ${ }^{25}$ and that a significant proportion of patients presenting with a LACS show cortical lesions on cerebral imaging. ${ }^{26}$ In our analysis, overlap of a LACS with other stroke syndromes persisted even when we considered only supratentorial small subcortical infarcts. As clinical classifications are frequently used for patient description and for selection for stroke trials, it is important to recognise this limitation in the accuracy of clinical classification of stroke subtypes. Apparently, patient selection for examination of therapeutic interventions targeted at specific stroke subtypes should be based on DWI.

Clearly, the retrospective design of our study imposes some limitations and the investigated cohort is not representative of the overall stroke population. Referral of patients to MRI was based solely on the discretion of the treating neurologist and may therefore have been prone to selection bias regarding age, stroke severity, or results from other examinations. However, it is the policy of our department to routinely obtain MRI in all stroke patients, and there were no specified guidelines to exclude patients from routine MRI other than those given in the Methods section. All ischaemic stroke patients who underwent MRI also had DWI sequences. The retrospective classification of patients according to the OCSP may have been influenced by the quality of the documentation of the patients' symptoms and signs, but a systematic bias towards a specific clinical syndrome seems unlikely.

For a targeted diagnostic procedure and specific secondary prevention, a clear association between infarct patterns and aetiology is desirable. In this context, small vessel disease and related risk factors, such as hypertension, have been implicated as the major causes of lacunar stroke. Hypertension is a predominant risk factor in our cohort of patients. Reported frequency rates of hypertension in patients with lacunar stroke vary from $24 \%$ to $97 \%$, with a mean of approximately $70 \%,{ }^{27}$ which is similar to our results. Not unexpectedly, we found hypertension to be especially prevalent in the subgroup of patients with an overall imaging pattern suggestive of microangiopathy. 
Table 1 Characteristics of patients with multiple $v$ single DWI lesions and of patients with a microangiopathy related imaging pattern compared to those without ("$p<0.05$; ${ }^{* *} p<0.001$ )

\begin{tabular}{|c|c|c|c|c|}
\hline & $\begin{array}{l}\text { Multiple DWI lesions } \\
\text { in different } \\
\text { vascular territories }\end{array}$ & $\begin{array}{l}\text { Single DWI lesion } \\
\text { or two lesions due } \\
\text { to occlusion of the } \\
\text { same small vessel }\end{array}$ & $\begin{array}{l}\text { Microangiopathy } \\
\text { related } \\
\text { imaging pattern }\end{array}$ & $\begin{array}{l}\text { Imaging not } \\
\text { suggestive of } \\
\text { microangiopathy }\end{array}$ \\
\hline Number of patients & 9 & 84 & 36 & 57 \\
\hline Age, years (range) & $55.9(38-70)^{*}$ & $66.7(28-89)$ & $71.8(47-88)^{\star *}$ & $61.9(28-89)$ \\
\hline \multirow{2}{*}{ Gender } & 8 men & 55 men & 19 men & 44 men \\
\hline & 1 woman & 29 women & 17 women* & 13 women \\
\hline \multirow[t]{3}{*}{ Clinical syndrome } & 3 LACS & 38 LACS & 12 LACS & 29 LACS \\
\hline & 6 POCS & 31 POCS & 14 POCS & 23 POCS \\
\hline & & $\begin{array}{l}14 \text { PACS } \\
1 \text { TACS }\end{array}$ & 10 PACS & 4 PACS \\
\hline Hypertension & $7(77.8 \%)$ & 60 (71.4\%) & $31(86.1 \%)^{*}$ & $36(63.2 \%)$ \\
\hline Hypercholesterolaemia & $5(55.6 \%)$ & $47(55.9 \%)$ & $24(66.7 \%)$ & $28(49.1 \%)$ \\
\hline Diabetes mellitus & $3(33.3 \%)$ & $27(32.1 \%)$ & $14(38.9 \%)$ & $16(28.1 \%)$ \\
\hline Smoking & $5(55.6 \%)$ & $21(25.0 \%)$ & $10(27.8 \%)$ & $26(45.6 \%)$ \\
\hline Large artery disease & $4(44.4 \%)$ & $37(44.0 \%)$ & $16(44.4 \%)$ & 25 (43.9\%) \\
\hline $\begin{array}{l}\text { Possible source of cardiac } \\
\text { embolism }\end{array}$ & $5(55.6 \%)^{*}$ & $20(23.8 \%)$ & $6(16.7 \%)$ & 19 (33.3\%) \\
\hline
\end{tabular}

DWI offers the advantage of differentiating acute from chronic small subcortical and brainstem ischaemic lesions with unprecedented accuracy. Therefore, DWI has been claimed to have the potential to improve early diagnosis of stroke aetiology according to the TOAST criteria, especially by identifying multiple acute lesions in different vascular territories. ${ }^{1728}$ However, the assumption that such lesions are strongly suggestive of an embolic aetiology has been questioned recently by a report of 10 patients with multiple DWI lesions in whom a cardioembolic source could only be identified in one patient. ${ }^{18}$ In contrast to this observation, we found a possible cardioembolic source in five out of nine individuals $(55.6 \%)$ with multiple small acute DWI lesions in truly different vascular territories. This may be explained in part by the fact that our study included data from TEE and/or TTE in all our patients with multiple DWI lesions, whereas Chowdhury et $a l^{18}$ obtained TTE in only three of their patients. In addition, these authors overlooked a mean interval of 17.7 days (range 2-45 days) from the onset of stroke symptoms to MRI for the identification of multiple lesions on DWI and noted that the lesions appeared to have occurred consecutively rather than simultaneously. As we analysed individuals within the first 7 days of stroke, multiple lesions in our patients are likely to have occurred simultaneously or at least within a short time interval only.

Various issues have to be considered regarding our observation of a rather high rate of possible cardioembolic pathologies and of large artery disease in this sample selected for small subcortical infarcts. Some of the detected cardiac pathologies presumably have a rather low probability of causing cerebral emboli, and even atrial fibrillation may not have been causally related to our patients' strokes. Yet the fact that small subcortical infarcts may indeed be due to an embolic aetiology cannot be dismissed..$^{15}$ 29-32 Furthermore, in attempting to optimise the prevention of further strokes, it appears plausible to appropriately treat all conditions with a high cardioembolic risk even if the association with the index stroke is not absolutely certain.

Whether carotid artery stenosis is causative or coincidental in lacunar stroke is a matter of ongoing debate. ${ }^{33}$ Large trials have reported a milder degree of ICA stenosis in patients with lacunar stroke, ${ }^{34}{ }^{35}$ which may suggest that large artery disease is coincidental in lacunar stroke. However, another study has found a higher incidence of ICA stenosis on the ipsilateral side in lacunar stroke, ${ }^{36}$ thereby suggesting a causal relationship. In our study, the severity of large artery stenosis was mild in most cases. We found only three patients with ICA stenosis $>50 \%$ and one patient with intracranial ICA stenosis in a total of 34 patients with a small subcortical lesion in the ICA territory.

In conclusion, MRI with the addition of DWI provides important information regarding stroke subtype and aetiology. Concomitant morphological features as proposed in our study may help to further differentiate true small vessel disease from other stroke subtypes. In this regard, the impact of age related cerebral white matter changes on vascular events is currently under investigation in a prospective multicentre trial. ${ }^{20}$ Large artery disease and potential sources of cardiac embolism may be not only coincidental but also causally related to small subcortical infarctions, thus underscoring the need for complete diagnostic evaluation in all stroke patients irrespective of their clinical presentation.

\section{ACKNOWLEDGEMENTS}

We thank P Ofner, Institute for Medical Informatics, Statistics and Documentation, Graz Medical University, and E Flooh, Department of Neurology, Graz Medical University, for skilful assistance with statistical calculations.

\section{Authors' affiliations}

T Seifert, C Enzinger, M K Storch, G Pichler, K Niederkorn, F Fazekas, Department of Neurology, Medical University Graz, Graz, Austria F Fazekas, Departments of Neurology and Radiology, Division of Neuroradiology, Medical University Graz, Graz, Austria

Competing interests: none declared

\section{REFERENCES}

1 Landi G, Cella E, Boccardi E, et al. Lacunar versus non-lacunar infarcts: pathogenetic and prognostic differences. J Neurol Neurosurg Psychiatry 1992; 55:441-5.

2 Fisher CM. Lacunes. Small, deep cerebral infarcts. Neurology 1965; 15:774-84

3 Fisher CM. The arterial lesions underlying lacunes. Acta Neuropathol (Berl) 1969:12:1-15.

4 Fisher CM, Caplan LR. Basilar artery branch occlusion: a cause of pontine infarction. Neurology 1971;21:900-5.

5 Fisher CM. Capsular infarct: the underlying vascular lesions. Arch Neurol 1979;36:65-73.

6 Fisher CM. Lacunar stroke and infarcts: a review. Neurology 1982;32:871-6.

7 Lodder J, Bamford JM, Sandercock PAG, et al. Are hypertension or cardiac embolism likely causes of lacunar infarction? Stroke 1990;21:375-81.

8 Boiten J, Lodder J. Lacunar infarcts. Pathogenesis and validity of the clinical syndromes. Stroke 1991;22:1374-8.

9 Bamford JM. Classical lacunar syndromes. In: Bogousslavsky J, Caplan L, eds. Stroke syndromes. Cambridge, UK: Cambridge University Press, 2001:583-9. 
10 Bamford JM, Sandercock P, Dennis M, et al. Classification and natural history of clinically identifiable subtypes of cerebral infarction. Lancet 1991;337:1521-6.

11 Adams HP, Bendixen BH, Kappelle $\sqcup$, et al. Classification of subtype of acute ischemic stroke. Definitions for use in a multicenter clinical trial. Stroke 1993;24:35-41.

12 Arboix A, Marti-Vilalta JL, Pujol J, et al. Lacunar cerebral infarct and nuclear magnetic resonance. Eur Neurol 1990;30:47-51.

13 Augustin M, Bammer R, Simbrunner J, et al. Diffusion-weighted imaging of patients with subacute cerebral ischemia: comparison with conventional and contrast-enhanced MR imaging. AJNR Am J Neuroradiol 2000;21:1596-1602.

14 Gass A, Ay H, Szabo K, et al. Diffusion-weighted MRI for the "small stuff": the details of acute cerebral ischemia. Lancet Neurol 2004;3:39-45.

15 Ay H, Oliveira-Filho J, Buonnanno FS, et al. Diffusion weighted imaging identifies a subset of lacunar infarction associated with embolic source. Stroke 1999;30:2644-50.

16 Baird AE, Lövblad KO, Schlaug G, et al. Multiple acute stroke syndrome. Marker of embolic disease? Neurology 2000;54:674-8.

17 Engelter ST, Wetzel SG, Radue EW, et al. The clinical significance of diffusion weighted MR imaging in infratentorial strokes. Neurology 2004;62:574-80.

18 Chowdhury D, Wardlaw JM, Dennis MS. Are multiple acute small subcortical infarctions caused by embolic mechanism? J Neurol Neurosurg Psychiatry 2004;75:1416-20.

19 Fazekas F, Chawluk JB, Alavi A, et al. MR signal abnormalities at 1.5 T in Alzheimer's dementia and normal aging. AJNR Am J Neuroradiol 1987;8:421-6.

20 Pantoni L, Basile AM, Pracucci G, et al. Impact of age-related cerebral white matter changes on the transition to disability - the LADIS study: rationale, design and methodology. Neuroepidemiology 2005;24:51-62.

21 de Bray JM, Glatt B. Quantification of atheromatous stenosis in the extracranial internal carotid artery. Cerebrovasc Dis 1995;5:414-26.

22 Perscheron G. Arteries of the human thalamus. II. Arteries and paramedian thalamic territory of the communicating basilar artery. Rev Neurol (Paris) $1976 ; 132: 309-24$.
23 Kumral E, Evyapan D, Balkir K, et al. Bilateral thalamic infarction. Clinical, etiological and MRI correlates. Acta Neurol Scand 2001;103:35-42.

24 Gerraty RP, Parsons MW, Barber AB, et al. Examining the lacunar hypothesis with diffusion and perfusion magnetic resonance imaging. Stroke 2002;33:2019-24.

25 Mead GE, Lewis SC, Wardlaw JM, et al. How well does the Oxfordshire Community Stroke Project classification predict the site and size of the infarct on brain imaging. J Neurol Neurosurg Psychiatry 2000;68:558-62.

26 Mead GE, Lewis SC, Wardlaw JM, et al. Should computed tomography appearance of lacunar stroke influence patient management? J Neurol Neurosurg Psychiatry 1999;67:682-4.

27 You R, McNeil JJ, O'Malley HM, et al. Risk factors for lacunar infarction syndromes. Neurology 1995;45:1483-7.

28 Lee LJ, Kidwell CS, Alger J, et al. Impact on stroke subtype diagnosis of early diffusion-weighted magnetic resonance imaging and magnetic resonance angiography. Stroke 2000;31:1081-9.

29 Arboix A, Padilla I, Massons J, et al. Clinical study of 222 patients with pure motor stroke. J Neurol Neurosurg Psychiatry 2001;71:239-42.

30 Jung DK, Devuyst G, Maeder $P$, et al. Atrial fibrillation with small subcortical infarcts. J Neurol Neurosurg Psychiatry $2001 ; 70: 344-9$.

31 Yonemura K, Kimura K, Minematsu K, et al. Small centrum ovale infarcts on diffusion-weighted magnetic resonance imaging. Stroke 2002;33:1541-4.

32 Baumgartner RW, Sidler C, Mosso M, et al. Ischemic lacunar stroke in patients with and without potential mechanism other than small-artery disease. Stroke 2003;34:653-9.

33 Devuyst G, Bogousslavsky J. The fall and rise of lacunar infarction with carotid stenosis. Stroke 2003;34:1409-11.

34 Boiten J, Rothwell PM. Slattery J, et al. Ischemic lacunar stroke in the European Carotid Surgery Trial: risk factors, distribution of carotid stenosis, effect of surgery and type of recurrent stroke. Cerebrovasc Dis 1996;6:281-7.

35 Inzitari D, Eliasziw M, Sharpe BL, et al. Risk factors and outcome of patients with carotid artery stenosis presenting with lacunar stroke. Neurology 2000;54:660-6.

36 Tejada J, Díez-Tejedor E, Hernández-Echebarría L, et al. Does a relationship exist between carotid stenosis and lacunar infarction? Stroke 2003;34:1404-11. 